\title{
Workstation-oriented distribution optimization of shipbuilding materials
}

\author{
Gang Chen ${ }^{1}$, Yan Jiang ${ }^{2,}$, Xing Sheng ${ }^{1}$, Jingqian $\mathrm{Wang}^{2}$, and Hui $\mathrm{Jia}^{2}$ \\ ${ }^{1}$ Shanghai Waigaoqiao Shipbuilding Co., Ltd., Shanghai 200137, China \\ ${ }^{2}$ College of Shipbuilding Engineering, Harbin Engineering University, Harbin 150001, China
}

\begin{abstract}
The optimization of material distribution is of great importance on shipbuilding project, which determines whether the production capacity of the ship is fully embodied. A workstation-oriented material distribution problem is formulated with reference to the production characteristics of shipyards. This problem can be considered as a complex vehicle routing problem (VRP) with capacity constraints, time windows and multiple distribution centers. In order to minimize the impact of distribution problems on production, a multi-population genetic algorithm (MPGA) that can minimize the sum of earliness and tardiness penalties is proposed in this paper. The proposed algorithm looks for near-optimal solutions for assigning distribution tasks and optimizing vehicle routing. Then, the evaluation of the solutions generated with MPGA is achieved with a priority-based heuristic algorithm. Simulation results of different cases show that the proposed MPGA allows logistics distribution system to operate more efficiently and solutions can be improved by $71 \%$ on average compared to those obtained with the traditional priority rule method.
\end{abstract}

\section{Introduction}

Material preparation is a prerequisite for shipbuilding. The refined production management under the modern shipbuilding mode puts forward higher requirements for the logistics and distribution of shipbuilding materials. Considering the characteristics of ship construction, a workstation-oriented material distribution method is usually adopted in shipyards. In practice, there are four main factors involved in the distribution of shipbuilding materials: (i) from which distribution center to start, (ii) which vehicle to designate, (iii) what vehicle route to use, and (iv) when delivered to the workstation. The workstation-oriented distribution problem of shipbuilding materials is a complex vehicle routing problem (VRP).

Jiang $\mathrm{Li}$ et al.[1] proposed a material delivery plan centering on the workstation in discrete manufacturing workshop. The materials are classified according to the demand of the workstations. They researched the material distribution optimization with hard time windows from the point of material storage and delivery. Chen et al.[2] introduced the concept of fuzzy time window into VRP. They analyzed several optimization objectives, such as fleet size, average customer satisfaction, transportation distance and queuing time. Then, the problem was solved by using a genetic algorithm. Taillard et al.[3] modeled the

\footnotetext{
* Corresponding author: jeangyan@hrbeu.edu.cn
} 
VRP with backhauls and soft time windows, and applied a tabu search heuristic to solve the problem.

Baños et al.[4] studied material distribution from the perspective of balancing the distribution path length and vehicle load, and suggested a simulated annealing-based parallel multi-objective approach. In view of the uncertain factors in material distribution, Li Jinhang et al.[5] put forward an opportunity constraint model under the condition of much fuzzy information. In order to minimize the transportation distance, an improved genetic algorithm was used to solve the model. Considering the change of material demand caused by the production fluctuations, Yan Zhengfeng et al.[6] established a path optimization model of workshop logistics based on fuzzy soft time windows, and solved the model with dynamic programming simulated annealing algorithm.

The rest of the paper is organized as follows. The following section gives a precise description to the workstation-oriented distribution problem of shipbuilding materials. Moreover, a mathematical model for the optimization problem is presented. In Section 3, the implementation of the proposed multi-population genetic algorithm (MPGA) is described. Section 4 presents the design of numerical experiments for evaluating the performance of MPGA and the corresponding results. Finally, the associated conclusion is presented in Section 5.

\section{Workstation-oriented distribution problem of shipbuilding materials}

\subsection{Problem description}

As shown in figure 1, there are $l$ vehicles (whose carrying capacity is C), $m$ distribution centers and $n$ workstations in the construction area of a shipyard. The vehicle is responsible for distributing shipbuilding materials from distribution centers to each workstation. For distribution task $i$, the time window $\left(L T_{i}, H T_{i}\right)$ is known and the time point when materials are delivered to the workstation is set as $R T_{i}$. When $R T_{i}<L T_{i}$, there is a earliness penalty. While $R T_{i}>H T_{i}$, it will have a tardiness penalty. To ensure the continuous and effective production, it's necessary to find the optimal vehicle scheduling plan such that the sum of earliness and tardiness penalties is minimized.
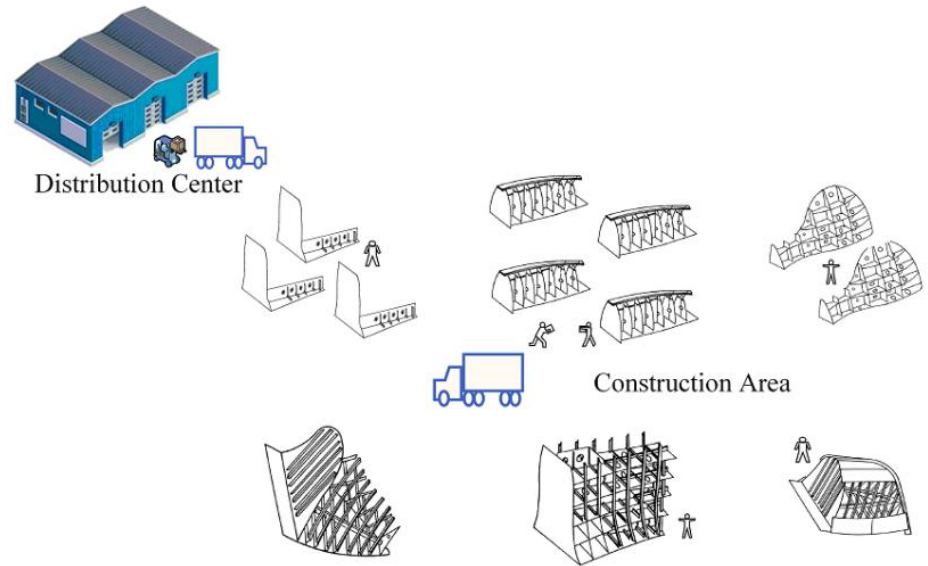

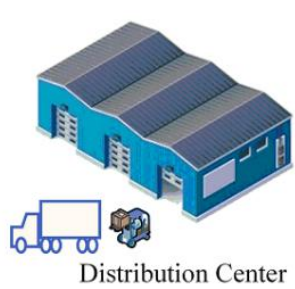

Distribution Center

Fig. 1. Schematic diagram of the distribution of shipbuilding materials.

In order to simplify the research, the following rules have been formulated :

- When delivery starts, the vehicle is dispatched from the distribution center. 
- The vehicle can simultaneously undertake multiple distribution tasks in one distribution list as long as its carrying capacity is not exceeded.

- After all materials have been delivered to the corresponding workstations, the vehicle must return to the same distribution center it dispatched from and starts the next distribution cycle.

- The materials required by a workstation can be delivered from any distribution center.

So whether the time windows of a set of distribution tasks can be met is dependent on (1) how distribution tasks are grouped into distribution lists, (2) how distribution lists are assigned to and sequenced by vehicles, and (3) how vehicles are routed to deliver materials of each distribution list.

\subsection{Model formulation}

According to the problem description, we introduce some related notations.

$V \quad$ set of vehicles $(V=\{1, \ldots, l\})$

$S \quad$ set of distribution tasks $(S=\{1, \ldots, N\})$

$L \quad$ set of all feasible distribution lists

$K$ set of positions where a distribution list can be scheduled of vehicle $v \in V$

$C$ carrying capacity of vehicles

$\left(L T_{i}, H T_{i}\right) \quad$ lower and upper limits of the delivery time of distribution task $i \in S$

$c_{i} \quad$ storage volume of the requested items in distribution task $i \in S$

$R T_{\mathrm{i}}$ receive time of distribution task $i \in S$

$p t_{i}$ processing time of distribution task $i \in S$

$c_{k v}$ completion time of distribution list scheduled at position $k \in K$ of vehicle $v \in V$

$E_{i} \quad$ earliness time of distribution task $i \in S$

$T_{i} \quad$ tardiness time of distribution task $i \in S$

$\alpha \quad$ earliness penalty per unit time

$\beta$ tardiness penalty per unit time

$a_{i j} \quad$ variable for indicating whether distribution task $i \in S$ is included in distribution list $j \in L\left(a_{i j}=1\right)$ or $\operatorname{not}\left(a_{i j}=0\right)$

$x_{i k v}$ variable for indicating whether distribution task $i \in S$ is allocated to the distribution list scheduled at position $k \in K\left(x_{i k v}=1\right)$ or not $\left(x_{i k v}=0\right)$ of vehicle $v \in V$

$y_{j k v}$ variable for indicating whether distribution list $j \in L$ scheduled at position $k \in K\left(y_{j k v}=1\right)$ or not $\left(y_{j k v}=0\right)$ of vehicle $v \in V$

$r_{z i} \quad$ variable for indicating whether another distribution task $z \in S$ is delivered before task $i \in S\left(r_{z i}=1\right)$ or not $\left(r_{z i}=0\right)$

The problem can then be formulated as follows.

$\min \quad \sum_{\mathrm{i} \in \mathrm{S}}\left(\alpha E_{i}+\beta \mathrm{T}_{i}\right)$

s.t.

$$
\begin{aligned}
& \sum_{j \in L} y_{j k v} \leq 1, \forall k \in K, v \in V \\
& \sum_{v \in V} \sum_{k \in K} y_{j k v}=1, \forall j \in L \\
& \sum_{j \in L} a_{i j} y_{j k v}=x_{i k v,}, \forall i \in S, k \in K, v \in V
\end{aligned}
$$




$$
\begin{aligned}
& \sum_{j \in L} a_{i j}=1, \forall i \in S \\
& \sum_{i \in S} c_{i} \cdot a_{i j} \leq C, \forall j \in L \\
& E_{i}=\max \left\{0, L T_{i}-R T_{i}\right\}, \forall i \in S \\
& T_{i}=\max \left\{0, R T_{i}-H T_{i}\right\}, \forall i \in S \\
& \sum_{i \in S} p t_{i} \cdot a_{i j} \cdot y_{j k v} \leq c t_{1 v}, \forall j \in L, v \in V \\
& \mathrm{RT}_{\mathrm{i}}=\sum_{\mathrm{z} \in \mathrm{S}} \mathrm{pt}_{\mathrm{z}} \cdot \mathrm{x}_{\mathrm{zkv}} \cdot \mathrm{r}_{\mathrm{zi}}, \mathrm{k}=1, \forall \mathrm{v} \in \mathrm{V} \\
& R T_{i}=c t_{(k-1) v}+\sum_{z \in S} p t_{z} \cdot x_{z k v} \cdot r_{z i}, \forall k \in K \backslash\{1\}, v \in V \\
& \mathrm{a}_{\mathrm{ij}}, \mathrm{x}_{\mathrm{ikv}}, \mathrm{y}_{\mathrm{jkv}}, \mathrm{r}_{\mathrm{zi}} \in\{0,1\}, \forall \mathrm{i}, \mathrm{z} \in \mathrm{S}, \mathrm{j} \in \mathrm{L}, \mathrm{k} \in \mathrm{K}, \mathrm{v} \in \mathrm{V}
\end{aligned}
$$

The objective function (1) minimizes the sum of earliness and tardiness penalties. Constraints (2) guarantee that at most one distribution list is scheduled at every position of each vehicle, while equation (3) ensures that each distribution list is performed. Equation (4) represents that the consistency among distribution tasks and distribution lists. Each distribution task can not be divided which is obtained by meeting restrictions (5). The carrying capacity of the vehicle is taken into account by satisfying equation (6). The earliness and tardiness time can be evaluated according to formulas (7) and (8) respectively. Inequality (9) is necessary to determine the completion time of the distribution list scheduled at position 1 of vehicle $v$. Equations (10) and (11) calculate the receive time of distribution tasks.

It is obvious that the problem is $N P$-hard [7]. Only if the problem size is small, can it be solved accurately. Thus, this paper suggests a multi-population genetic algorithm to solve the model.

\section{Multi-population genetic algorithm}

\subsection{General principle}

\begin{tabular}{|c|}
\hline Algorithm 1 classic genetic algorithm \\
\hline $1:$ start \\
\hline 2: $\quad \mathrm{t}=0$ \\
\hline 3: initialize population $\mathrm{P}(\mathrm{t})$ \\
\hline 4: evaluate $\mathrm{P}(\mathrm{t})$ \\
\hline 5: while (termination condition is not met) do \\
\hline 6: $\quad$ Selection \\
\hline 7: $\quad$ Crossover \\
\hline
\end{tabular}

Genetic algorithm was first proposed by Holland [8], which was inspired by the notion of biological evolution. Typically, it processes a set of mathematical objects into a constant length string which is called chromosome [9]. Through the continuous evolution of chromosome population, genetic algorithm can converge to the fittest individual eventually to obtain the optimal or satisfactory solution. The classic genetic algorithm is summarized in table 1.

Table 1. Classic genetic algorithm. 


\begin{tabular}{|ll|}
\hline $8:$ & Mutation \\
\hline $9:$ & recombine $\mathrm{P}(\mathrm{t})$ to produce subpopulation $\mathrm{C}(\mathrm{t})$ \\
\hline $10:$ & evaluate $\mathrm{C}(\mathrm{t})$ \\
\hline $11:$ & Select $\mathrm{P}(\mathrm{t}+1)$ from $\mathrm{P}(\mathrm{t})$ and $\mathrm{C}(\mathrm{t})$ \\
\hline $12:$ & $\mathrm{t}=\mathrm{t}+1$ \\
\hline $13:$ & end \\
\hline $14:$ end \\
\hline
\end{tabular}

Although genetic algorithms have been successfully applied to many practical problems, there are still limitations, especially precocious convergence and bad local optimization ability. In order to improve the solution quality and accelerate the convergence, a multipopulation genetic algorithm (MPGA) is suggested. Figure 2 illustrates the structure of MPGA.

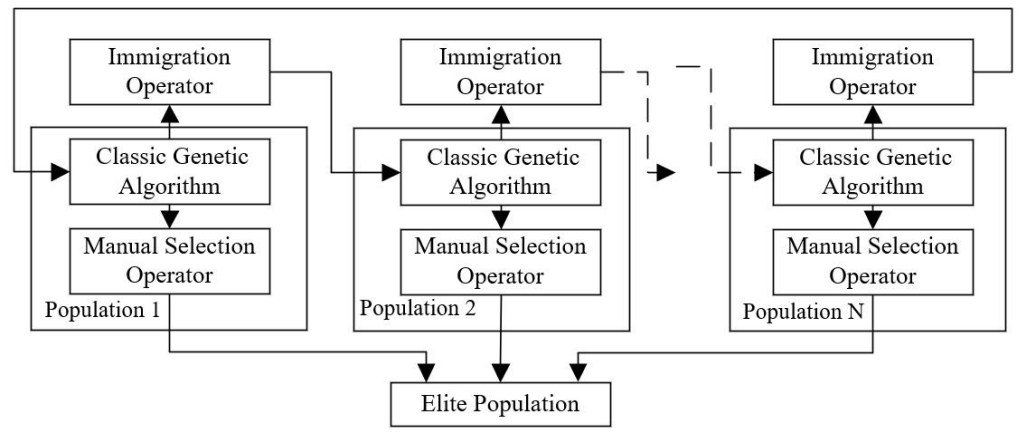

Fig. 2. Structural schematic diagram of the multi-population genetic algorithm.

\subsection{Adaption for the problem}

\subsubsection{Encoding}

Chromosome coding refers to the abstraction of a set of research objects into a string of specific symbols according to certain mechanism. A two-dimensional integer coding method is applied. For example, ten distribution tasks are required by workstations from two distribution centers with two vehicles each. One feasible solution encoded in a chromosome is shown in figure 3.

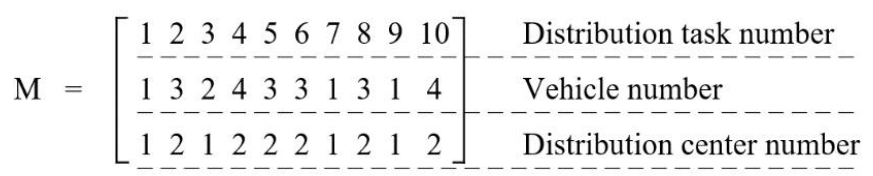

Fig. 3. A feasible chromosome encoding in the multi-population genetic algorithm.

\subsubsection{Fitness function}

Fitness function is the driving force of the evolution process of genetic algorithm. It is the basis of evaluating individuals in genetic algorithm. The larger the fitness function value is, the better the quality of solution is. According to the constraints of the problem, the individual fitness value of a feasible solution $\mathrm{i}$ is designed as

$$
\text { Fit }_{i}=1 / \sum_{i \in S}\left(\alpha E_{i}+\beta T_{i}\right)
$$




\subsubsection{Selection}

Selection operator selects chromosomes from the current generation to be copied into the next generation [10]. A stochastic universal sampling method is adopted. The probability of solution $i$ to be selected is

$$
p_{i}=F_{i t} / \sum F i t_{i}
$$

\subsubsection{Crossover and mutation}

A two-point crossover method is utilized in crossover operation. In this way, the parts between two points of two parent chromosomes are exchanged. The mutation operation makes each component in the chromosome has a probability (i.e. the mutation rate) to change its information. A single-point mutation method is used here.

\subsubsection{Immigration operator}

In MPGA, all populations are linked by immigration operators to achieve co-evolution. The immigration operators introduce the optimal individuals that have appeared in evolution process of each population into other populations at regular intervals. The specific operating rule is to replace the worst individual in target population with the best individual of source population.

\subsubsection{Manual selection operator and elite population}

In every generation, the best individual in each population is put into the elite population by manual selection operators. The elite population does not perform operations such as selection, crossover, and mutation, so as to ensure that the optimal individuals generated by each population are not destroyed or lost. Moreover, the minimum generation for optimal individual maintenance in elite population is used as the termination criterion of MPGA.

\section{Experiments}

Numerical experiments have been carried out to verify the performance of the suggested algorithm. In these experiments, a construction area layout simplified from the actual shipyard is considered. In figure 4, an exemplary visualization of this type of layout is depicted.

The construction area consists of 48 workstations, 2 distribution centers ( 2 vehicles for each), 4 horizontal aisles and 5 vertical aisles. Both the length of workstation and the width of aisle amount to one length unit (LU). The processing time of a distribution task is composed of setup time, travel time and unloading time [11]. For travel time, we assume that it takes $5 \mathrm{~s}$ for a vehicle to drive $1 \mathrm{LU}$. Moreover, it needs $5 \mathrm{~s}$ to unload an item. Each tour requires 3 minutes spent on administration and setup. 


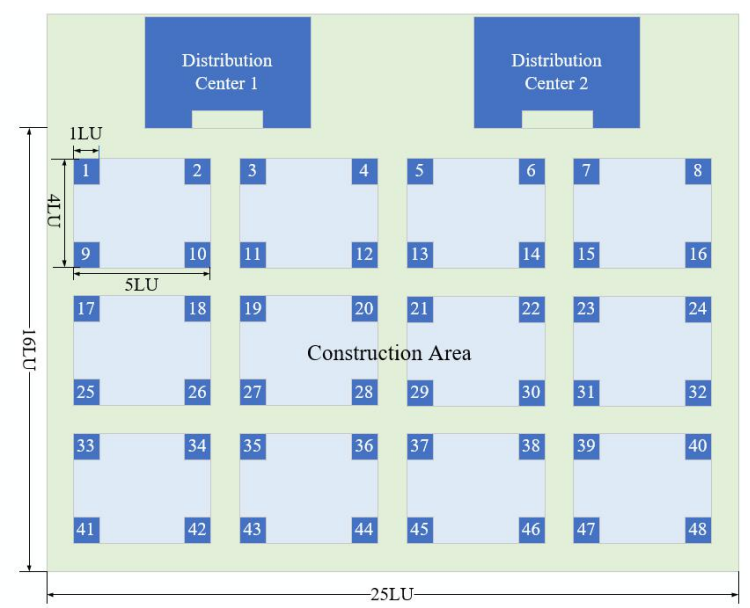

Fig. 4. Simplified layout of the construction area in shipyard.

In our numerical experiments, the set of instances considers 4 different number of distribution tasks $(\mathrm{N}=\{24,48,72,96\})$, where the number of items per task is uniformly distributed in $\{5,6, \ldots, 25\}$. The carrying capacity of vehicles is fixed to $45^{[12]}$. The delivery time for each task is chosen from the interval

$$
\left[0,2(1-\mathrm{MTCR}) \sum_{i \in S} p t_{i}^{s} / l\right], 0 \leq \mathrm{MTCR} \leq 1
$$

where $p t_{i}^{S}$ stands for the processing time of task $i(i \in S)$ if it is processed in a tour separately ${ }^{[13]}$. The values of modified traffic congestion rate (MTCR) considered are: 0.60 , 0.65 and 0.70 . The length of each time window is

$$
2(1-\mathrm{MTCR}) \sum_{i \in S} p t_{i}^{S} / N, 0 \leq \mathrm{MTCR} \leq 1
$$

As for routing strategy, the shortest path between any two workstations (or between distribution center and workstation) is adopted. Combining the penalty coefficient $(\alpha, \beta)$ which is set to $(0,1),(0.25,0.75)$, and $(0.5,0.5)$, we obtain a total of 36 problem classes, of which each has generated 50 instances.

To provide a method against which MPGA can be benchmarked, a priority-based heuristic algorithm is introduced. Under this algorithm, the selection of distribution center is determined by which one is closer to the workstation. Then all tasks dispatched from the same distribution center are sorted according to their delivery time in a non-decreasing order. According to this sequence, tasks are assigned to distribution lists based on the carrying capacity of vehicles. Figure 5 demonstrates the development of optimal values in the iterative processes of MPGA.

Table 2 describes the experimental results for object function value (obV), total earliness (ear) and total tardiness (tar) of the proposed algorithm. In addition, total processing time, i.e., makespan (mak) and vehicle utilization (uti) are presented. Moreover, the table depicts the improvement of object function value (imp) obtained by the evaluated algorithms. All entries in table 2 are the average solution values of the 50 implemented instances in each problem class. 


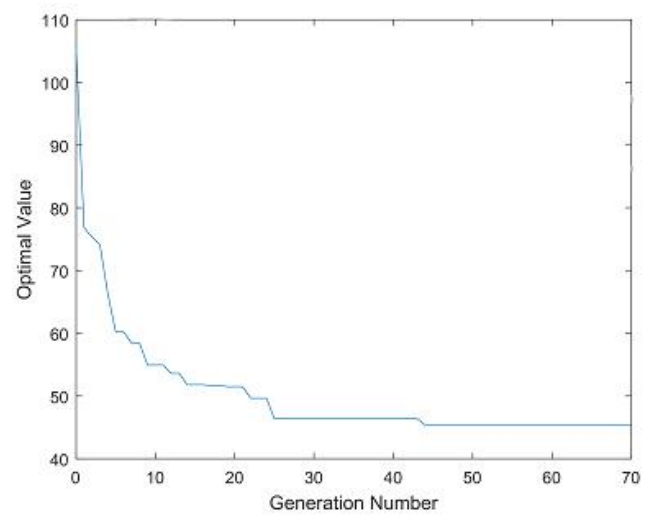

Fig. 5. Example for the optimization process of using MPGA to solve the instance (when $\mathrm{N}=48$, $\mathrm{MTCR}=0.65, \alpha=0.5, \beta=0.5$ ).

Table 2. The results of numerical experiments.

\begin{tabular}{|c|c|c|c|c|c|c|c|c|c|c|c|c|c|}
\hline \multirow[t]{2}{*}{$\mathrm{N}$} & MT & \multirow[t]{2}{*}{$(\alpha, \beta)$} & \multicolumn{5}{|c|}{ Priority-Based Heuristic Algorithm } & \multicolumn{6}{|c|}{ Multi-Population Genetic Algorithm } \\
\hline & $\mathrm{CR}$ & & $\mathrm{obV}$ & $\begin{array}{c}\text { ear } \\
(\mathrm{min}) \\
\end{array}$ & $\begin{array}{c}\operatorname{tar} \\
(\mathrm{min}) \\
\end{array}$ & $\begin{array}{l}\text { mak } \\
(\mathrm{min}) \\
\end{array}$ & $\begin{array}{l}\text { uti } \\
(\%) \\
\end{array}$ & $\mathrm{obV}$ & $\begin{array}{c}\text { ear } \\
(\mathrm{min}) \\
\end{array}$ & $\begin{array}{c}\operatorname{tar} \\
(\mathrm{min}) \\
\end{array}$ & $\begin{array}{l}\text { mak } \\
(\mathrm{min}) \\
\end{array}$ & $\begin{array}{l}\text { uti } \\
(\%) \\
\end{array}$ & $\begin{array}{l}\text { imp } \\
(\%) \\
\end{array}$ \\
\hline \multirow[t]{9}{*}{24} & 0.60 & $(0,1)$ & 9 & 41 & 9 & 29 & 73 & 0 & 36 & 0 & 27 & 76 & 100 \\
\hline & & $(0.25,0.75)$ & 18 & 37 & 12 & 30 & 74 & 4 & 15 & 0 & 28 & 75 & 78 \\
\hline & & $(0.5,0.5)$ & 24 & 38 & 10 & 29 & 75 & 6 & 11 & 1 & 28 & 77 & 74 \\
\hline & 0.65 & $(0,1)$ & 28 & 23 & 28 & 31 & 73 & 3 & 20 & 3 & 25 & 80 & 91 \\
\hline & & $(0.25,0.75)$ & 23 & 21 & 24 & 31 & 74 & 5 & 12 & 2 & 26 & 80 & 79 \\
\hline & & $(0.5,0.5)$ & 25 & 27 & 24 & 30 & 73 & 7 & 10 & 3 & 26 & 79 & 74 \\
\hline & 0.70 & $(0,1)$ & 44 & 13 & 44 & 29 & 74 & 9 & 10 & 9 & 24 & 85 & 80 \\
\hline & & $(0.25,0.75)$ & 38 & 11 & 47 & 31 & 74 & 12 & 7 & 14 & 25 & 85 & 67 \\
\hline & & $(0.5,0.5)$ & 27 & 13 & 42 & 29 & 74 & 10 & 7 & 14 & 25 & 84 & 62 \\
\hline \multirow[t]{9}{*}{48} & 0.60 & $(0,1)$ & 17 & 219 & 17 & 55 & 77 & 0 & 201 & 0 & 52 & 78 & 99 \\
\hline & & $(0.25,0.75)$ & 73 & 227 & 21 & 54 & 78 & 23 & 89 & 1 & 56 & 76 & 68 \\
\hline & & $(0.5,0.5)$ & 125 & 226 & 25 & 55 & 77 & 45 & 85 & 5 & 57 & 76 & 64 \\
\hline & 0.65 & $(0,1)$ & 68 & 135 & 68 & 55 & 77 & 4 & 117 & 4 & 49 & 83 & 94 \\
\hline & & $(0.25,0.75)$ & 70 & 122 & 53 & 54 & 77 & 22 & 69 & 6 & 50 & 81 & 69 \\
\hline & & $(0.5,0.5)$ & 89 & 115 & 64 & 55 & 77 & 35 & 56 & 14 & 50 & 82 & 61 \\
\hline & 0.70 & $(0,1)$ & 138 & 68 & 138 & 55 & 78 & 42 & 66 & 42 & 46 & 86 & 69 \\
\hline & & $(0.25,0.75)$ & 113 & 71 & 127 & 55 & 78 & 45 & 58 & 40 & 46 & 86 & 60 \\
\hline & & $(0.5,0.5)$ & 108 & 64 & 152 & 56 & 78 & 50 & 44 & 56 & 47 & 85 & 54 \\
\hline \multirow[t]{9}{*}{72} & 0.60 & $(0,1)$ & 28 & 580 & 28 & 80 & 79 & 0 & 549 & 0 & 75 & 81 & 100 \\
\hline & & $(0.25,0.75)$ & 162 & 578 & 24 & 77 & 79 & 59 & 227 & 2 & 84 & 77 & 64 \\
\hline & & $(0.5,0.5)$ & 316 & 598 & 33 & 80 & 79 & 110 & 205 & 14 & 87 & 77 & 65 \\
\hline & 0.65 & $(0,1)$ & 104 & 315 & 104 & 79 & 79 & 5 & 296 & 5 & 71 & 83 & 95 \\
\hline & & $(0.25,0.75)$ & 156 & 333 & 97 & 79 & 79 & 61 & 206 & 13 & 75 & 82 & 61 \\
\hline & & $(0.5,0.5)$ & 211 & 304 & 118 & 80 & 79 & 86 & 143 & 29 & 75 & 81 & 59 \\
\hline & 0.70 & $(0,1)$ & 272 & 145 & 272 & 80 & 79 & 92 & 152 & 92 & 67 & 87 & 66 \\
\hline & & $(0.25,0.75)$ & 249 & 159 & 279 & 79 & 79 & 120 & 149 & 110 & 68 & 87 & 52 \\
\hline & & $(0.5,0.5)$ & 219 & 157 & 280 & 79 & 79 & 116 & 109 & 124 & 68 & 86 & 47 \\
\hline \multirow[t]{3}{*}{96} & 0.60 & $(0,1)$ & 38 & 1073 & 38 & 105 & 80 & 0 & 1042 & 0 & 100 & 81 & 100 \\
\hline & & $(0.25,0.75)$ & 291 & 1101 & 21 & 100 & 80 & 113 & 438 & 4 & 113 & 77 & 61 \\
\hline & & $(0.5,0.5)$ & 553 & 1059 & 47 & 105 & 80 & 205 & 387 & 23 & 114 & 77 & 63 \\
\hline
\end{tabular}




\begin{tabular}{|cc|ccccc|cccccc|}
0.65 & $(0,1)$ & 164 & 572 & 164 & 104 & 80 & 7 & 556 & 7 & 94 & 84 & 95 \\
& $(0.25,0.75)$ & 266 & 619 & 149 & 103 & 80 & 108 & 367 & 22 & 99 & 82 & 59 \\
& $(0.5,0.5)$ & 353 & 547 & 159 & 103 & 80 & 159 & 261 & 57 & 100 & 81 & 55 \\
0.70 & $(0,1)$ & 404 & 294 & 404 & 102 & 80 & 120 & 307 & 120 & 88 & 88 & 70 \\
& $(0.25,0.75)$ & 369 & 295 & 394 & 101 & 80 & 180 & 288 & 143 & 89 & 87 & 51 \\
& $(0.5,0.5)$ & 354 & 266 & 443 & 103 & 79 & 206 & 205 & 207 & 91 & 86 & 42 \\
Average & & & & & & & & & & & & 71 \\
\hline
\end{tabular}

As expected, the object function value increases with an increasing number of distribution tasks. Furthermore, when the number of tasks is the same, the total earliness is reduced as MTCR increases, while the total tardiness increases. For problem classes with $\alpha=0$ and $\beta=1$, take no account of the impact of early delivery to production. For problem classes with $\alpha=0.25$ and $\beta=0.75$, the effect of delayed delivery on production is considered to be grater than early delivery. When $\alpha$ and $\beta$ are equal, it is considered that the effect of early delivery and delayed delivery on production is the same. The object function value gained by MPGA improves by an average of $71 \%$ over that of priority-based heuristic algorithm, which is a significant improvement in actual application.

\section{Conclusion}

In this work, we dealt with the workstation-oriented distribution problem of shipbuilding materials, which can be considered as a complex vehicle routing problem. Although many researchers have developed heuristic algorithms to solve vehicle routing problems, most of them are designed for specific problem classes and cannot be applied to this problem without the combination of production characteristics of shipyards. We proposed a multipopulation genetic algorithm that introduces immigration and manual selection operators to enhance local search capability and accelerate convergence. Simulation results demonstrate that the solutions generated by the proposed MPGA outperform those of the benchmark algorithm. The proposed MPGA increases the quality of solutions obtained by prioritybased heuristic algorithm by $71 \%$. It is worth noting that when the number of distribution tasks $(\mathrm{N})$ is small, the optimal solution can be obtained by MPGA generally. When $\mathrm{N}$ is relatively large, MPGA can only obtain an approximate solution. In this case, the optimization result can be improved by increasing the number of chromosome individuals and the minimum generation for optimal individual maintenance in elite population.

\section{Acknowledgements}

The authors would like to thank the editor and anonymous reviewers for their comments and suggestions. This research is partially supported by programs "Large Cruise Research and Development Project (2017)" and "Research on Key Common Technologies towards Smart Manufacturing in Shipbuilding Industry" funded by Ministry of Industry and Information Technology of the People's Republic of China (No. 2016543), and this paper is also supported by National Natural Science Foundation of China (No. 51679059).

\section{References}

1. Jiang L, Ding B and Zang X 2009 Workstation-oriented production logistics distribution optimization Comput. Integr. Manuf. 154 700-5.

2. Cheng R, Gen M and Tozawa T 1995 Vehicle routing problem with fuzzy due-time using genetic algorithms J. Jpn. Soc. Fuzz. 7 1050-61 
3. Duhamel C, Potvin J and Rousseau J 1997 A tabu search heuristic for the vehicle routing problem with backhauls and time windows INFORMS 31 49-59

4. Baños R, Ortega J, Gil C, Fernández A and Toro F 2013 A simulated annealing-based parallel multi-objective approach to vehicle routing problems with time windows Expert. Syst. Appl. 40 1696-707

5. Li J, Huang G and Jia Y 2011 Vehicle routing problem in material distribution under condition of much fuzzy information J. Mech. Eng. 47 124-31

6. Yan Z, Mei F and Ge M 2015 Path optimization method of workshop logistics based on fuzzy soft time windows Comput. Integr. Manuf. 21 2760-67

7. Scholz A, Schubert D and Wäscher G 2017 Order picking with multiple pickers and due dates -simultaneous solution of order batching, batch assignment and sequencing, and picker routing problems Eur. J. Oper. Res. 263 461-78

8. Holland J H 1975 Adaptation in natural and artificial systems Q. Rev. Biol. 6 126-37

9. Ahmadi M R 2018 Cutoff grade optimization in open pit mines using genetic algorithm Resour. Policy. 55 184-91

10. Pan C H, Shih P H and Wu M H 2015 Order batching in a pick-and-pass warehousing system with group genetic algorithm Omega-Int. J. Manage. S. 57 238-48

11. Henn S and Schmid V 2013 Metaheuristics for order batching and sequencing in manual order picking systems Comput. Ind. Eng. 66 338-51

12. Menéndez B, Bustillo M, Pardo E G and Duarte A 2017 General variable neighborhood search for the order batching and sequencing problem Eur. J. Oper. Res. 263 82-93

13. Elsayed E A, Lee M K, Kim S and Scherer E 1993 Sequencing and batching procedures for minimizing earliness and tardiness penalty of order retrievals Int. J. Prod. Res. 31 727-38 